\title{
ON TOPOLOGICAL INTERPRETATION OF QUANTUM NUMBERS
}

\author{
S.A.Bulgadaev" \\ Landau ITP RAS, 117334, Moscow, Kosyghin street, 2
}

\begin{abstract}
It is shown, how one can define vector topological charges for topological exitations of non-linear $\sigma$-models on compact homogeneous spaces $T_{G}$ or $G / T_{G}$ (where $G$ is a simple compact Lie group and $T_{G}$ is its maximal commutative subgroup). Explicit solutions for some cases, their energies and interaction of different topological charges are found. A possibility of the topological interpretation of the quantum numbers of groups and particles is discussed.
\end{abstract}

\footnotetext{
${ }^{1}$ This work is supported by RFBR grants $96-02-17331$ and $96-1596861$
} 


\section{Introduction}

The discovery of new topologically stable solutions [1-4] has revived the old, ascending to antiquity, hypothesis about a topological nature of simplest particles. For example, Descartes offered vortex model of magnetism [5], while lord Kelvin has putted forward a conjecture that atoms can be represented as knotted configurations of "ether" [6]. Though it has appeared that both conjectures are non correct they were very fruitful and have stimulated the investigation of these phenomena as well as development of the vorticity and of the knot theories [7] and their applications in different regions of physics from hydrodynamics till polymers [8-11]. An advantage of this idea in comparison with usual, considering simplest particles as a pointlike, structureless objects, is that it offeres an obvious image of elementary particles. Later, analogous ideas have renewed periodically in connection with different events in history of physics, (the most succes they achieved in condensed matter physics, see for example [11]), but, in the field theory, they usually have been based on one or another topological invariant and for this reason a set of topological charges, offered by them, were not reach enough for complete description of real particles with internal symmetries (there is a huge number of literature on this subject, we note here only [12] for further information).

A modern physics has many common properties with physics of second part of XIX century with condensate of various fields playing role of ether or others hypotetical liquids. For this reason a rebirth of hypothesis about topological origin of particles and their properties is very natural and it looks attractive from physical point of view, since it brings more deep understanding of the nature of particles and gives more obvious interpretation of their structure and quantum numbers in contrast with existing formal, purely Liealgebraic and structureless, description. To this one can add, that for this period a topology was strongly developed and adequate mathematical methods for description and investigation of various topological problems has been constructed (see for example [13]).

In order to this interpretation was possible, the following points are necessary:

1) the corresponding field theory must have degenerate vacuum, which forms some manifold $\mathcal{M}$ with nontrivial topology,

2) a theory must have solutions with nontrivial topology and finite (or logariphmic) energy, 
3) the set of possible topological charges must be the same as the set of possible quantum numbers.

The two first points are now fulfilled for such topological solutions as solitons, vortices, instantons and monopoles, but a third point remains incompleted, at least for first three exitations.

Usually quantum numbers of particles are determined by the weights of irreducible representations to which they belong. As is known the quantum numbers or weights of simple compact groups $G$ are connected with the maximal commutative subgroup $T_{G}$ of $G$ - the maximal abelian Cartan torus [14].

All possible weights of compact simple group form $n$-dimensional lattice $\mathbb{L}_{w}$, where $n$ is a rank of group $G$. Thus, the topological charges must also belong to the same lattice. Earlier it had been shown that solitons with topological isovectorial charges, belonging to the corresponding root, weight and dual root lattices $\mathbb{L}_{r}, \mathbb{L}_{w}, \mathbb{L}_{v}$ of groups $G$ exist in two-dimensional theories, generalizing sine-Gordon theory and connected with characters of groups with rank $n>1$ [15]. These charges can be related with homotopy group $\pi_{0}$ of vacuum configurations of these models, which form infinite discrete sets, coinciding with those lattices.

In general a possibility of existence of topological exitations in systems with degenerate vacuum, depends on a non-triviality of the homotopy groups $\pi_{i}(\mathcal{M})$ of vacuum space $\mathcal{M}$. For example, to usual vortices with integer topological charges $e \in \mathbb{Z}$ and one-dimensional instantons [16] corresponds $\pi_{1}\left(S^{1}\right)=\mathbb{Z}$, while the two-dimensional instantons correspond to $\pi_{2}\left(S^{2}\right)=\mathbb{Z}$ [4, 13]. As is shown in [17], for obtaining topological charges belonging to weight lattice of compact simple Lie group $G$ it is necessary to consider in case of vortices a torus $T_{G}$ and in a case of instantons a more special homogeneous space of $G$, a flag space $F_{G}=G / T_{G}$.

In this paper we continue consideration of these manifolds $\mathcal{M}$. It will be shown how one can define corresponding vector topological charges and some explicit solutions with such topological charges will be found. We will deal mainly with non-linear $\sigma$-models on manifolds $\mathcal{M}$, since they are effective theories for large class of models having $\mathcal{M}$ as their vacuum manifolds. We also discuss conditions under which quantum numbers of groups admit topological interpretation. 


\section{Manifolds with vectorial homotopy group $\pi_{1}$}

The study of the manifolds with vectorial (i.e. with rank $n>1$ ) homotopy group it is convenient to begin with manifolds with vectorial $\pi_{1}(\mathcal{M})$ groups. For our purposes it will be enough to consider only spaces with abelian $\pi_{1}$, since the corresponding topological charges must commute with each other. We also confine ourselves by free homotopic groups for simplicity. Though their properties are simple and well known we need discus them with a special attention paid to their vector structure, since it help us under consideration of spaces with vectorial $\pi_{2}$. The simplest generalization of circle

$$
S^{1}=e^{2 \pi i \phi}, \quad 0 \leq \phi \leq 1
$$

is a torus $T^{n}$, which equals to the direct product of $n$ circles

$$
T^{n}=\bigotimes_{i=1}^{n} S_{i}^{1} .
$$

The torus $T^{n}$ can be also represented as a coset space of $n$-dimensional euclidean space $R^{n}$

$$
T^{n}=\mathbb{R}^{n} / \mathbb{Z}^{n},
$$

where $\mathbb{Z}^{n}$ is $n$-dimensional simple cubic integer-valued lattice

$$
\mathbb{Z}^{n}=\bigoplus_{i=1}^{n} \mathbb{Z}_{i}
$$

A more general $n$-dimensional torus $T_{L}$ of rank $n$ can be defined as a factor

$$
T_{L}=\mathbb{R}^{n} / \mathbb{L}
$$

where $\mathbb{L}$ is some full $n$-dimensional lattice in $\mathbb{R}^{n}$

$$
\mathbb{L}=\sum_{i=1}^{n} n_{i} \mathbf{e}_{i}, n_{i} \in \mathbb{Z}_{i}, \mathbf{e}_{i} \in\left\{\mathbf{e}_{i}\right\}_{L},
$$

Here a set of linearly independent vectors $\left\{\mathbf{e}_{i}\right\}_{L}, i=1, \ldots, n$ forms a basis of lattice $\mathbb{L}$. For $T^{n}\left\{\mathbf{e}_{i}\right\}_{L}$ coincides with the canonical orthonormal basis

$$
\mathbf{e}_{i}=\left(0, . ., 0,1_{i}, 0, \ldots, 0\right), i=1, \ldots, n .
$$


A basis of any lattice can be choosed by many ways, all of them are related by modular transformations

$$
\left\{\mathbf{e}_{i}^{\prime}\right\}_{L}=M\left\{\mathbf{e}_{i}\right\}_{L}, \operatorname{det}(M)= \pm 1
$$

where matrices $M$ have integer-valued entries and for basises having the same orientation $\operatorname{det}(M)=1$. Usually the most convenient basis is the one having vectors with minimal possible norms.

The first homotopy group of torus $\pi_{1}\left(T^{n}\right)$, being the homotopical mapping classes of $S^{1}$ into $T^{n}$, is usually written as

$$
\pi_{1}\left(T^{n}\right)=\bigoplus_{i=1}^{n} \mathbb{Z}_{i}=\mathbb{Z}^{n},
$$

where $i$-th component describes mappings of $S^{1}$ into $i$-th circle of $T^{n}$. It is clear that mapping from different components cannot annihilate each other. The same expression is often used for other tori $T_{L}$. Then this form indicates only that coordinates of different homotopical classes are integer-valued in some basis, but does not contain any information about this basis. It means that one must introduce in $\pi_{1}\left(T_{L}\right)$ a vectorial structure.

To find out an explicit form of this basis one can take into account a quotient nature of $T_{L}$. Then it is desirable to choose it compatible with the vectorial structure of the covering space $\mathbb{R}^{n}$. The most natural way to do this is to conserve the euclidean vectorial structure of $\mathbb{R}^{n}$ and of $\mathbb{L}$, embedded into it. Then one comes to the next expression for $\pi_{1}\left(T_{L}\right)$

$$
\pi_{1}\left(T_{L}\right)=\mathbb{L}
$$

where now $\mathbb{L}$ contains in explicit form its basic vectors $\mathbf{e}_{i}$. This form can have more information than (7), since, except of integer-valuedness of homotopical classes $\left(n_{i} \in \mathbb{Z}_{i}\right)$, it contains geometrical characteristics of $T_{L}$. One need also to know metric and corresponding scalar product in space of topological charges, which may differ from that of covering space (see below). They will be very important for discussion of interaction of topological exitations with different topological charges.

Since to each basic vector $\mathbf{e}_{i}$ corresponds an elementary nontrivial homological cycle $\gamma_{i}$ of $T_{L}$, the corresponding homological group of cycles $H_{1}\left(T_{L}, \mathbb{Z}\right)$ of torus $T_{L}$ coincides with $\pi_{1}\left(T_{L}\right)$. This is a realization of the 
Gurevich theorem about isomorphism of first non-trivial homotopic and homological groups 13 . Consequently, $H_{1}\left(T_{l}, \mathbb{Z}\right)$ also has vectorial structure, which can be written as

$$
\gamma=\sum n_{i} \gamma_{i}, \gamma_{i}=\gamma_{i} \mathbf{e}_{i}, n_{i} \in Z
$$

There is also a cohomological group of 1 -forms $\delta \in H^{1}\left(T_{L}, \mathbb{R}\right)$, dual to $H_{1}\left(T_{L}, \mathbb{Z}\right)$, with basis $\left\{\boldsymbol{\delta}_{i}\right\}=\left\{\mathbf{s}_{i} d \phi^{i}\right\}$ and a convolution

$$
\left(\boldsymbol{\gamma}_{i} \circ \boldsymbol{\delta}_{k}\right)=\left(\mathbf{s}_{i} \mathbf{e}_{k}\right) \oint_{\gamma_{i}} d \phi^{k}=\delta_{i k}
$$

where $\left\{\mathbf{s}_{i}\right\}$ is a basis of dual lattice $\mathbb{L}^{*}$ and

$$
\left(\mathbf{s}_{i} \mathbf{e}_{k}\right)=\delta_{i k}
$$

here () means an euclidean scalar product.

Usually the topological charges $Q$ of $T^{n}$ are defined as a set of integer numbers, corresponding to the winding numbers around elementary cycles $\gamma_{i}$

$$
Q=\left(q_{1}, \ldots, q_{n}\right), q_{i} \in \mathbb{Z}_{i}
$$

The winding numbers can be written as integrals of 1 -forms $\delta_{k}$, dual to the cycles $\gamma_{i}$, in the parameter space of $T^{n}$

$$
q_{i}=\oint_{\gamma_{i}} d \phi=\oint_{\gamma_{i}} \sum_{k=1}^{n} n_{k} d \phi_{k}=n_{i}, d \phi=\sum_{k=1}^{n} n_{k} d \phi_{k}
$$

or

$$
q_{i}=\oint_{\gamma} d \phi_{i}=\sum_{k=1}^{n} n_{k} \oint_{\gamma_{k}} d \phi_{i}=n_{i}, \oint_{\gamma_{i}} d \phi_{k}=\delta_{i k}, \gamma=\sum_{k=1}^{n} n_{k} \gamma_{k}
$$

Here $\gamma$ and $d \phi$ are some closed contour and 1-form on $T^{n}$. Analogous expressions for $Q$ are usually used for any $T_{L}$. In this formal form all $Q$ have the similar, integer-valued, structure and all geometrical properties are hidden again in abstract basises of elementary cycles or 1-forms.

For obtaining vector topological charges, characterizing vectorial homotopic group, one can use any vector structure, mentioned above. For example, one can lift on the covering space $\mathbb{R}^{n}$ and define topological charges $\mathbf{Q}$ as 
line integrals of vector 1 -forms $d x^{k} \quad\left(\mathbf{x} \in \mathbb{R}^{n}\right)$ along preimage (or pull-back) curve $\gamma^{*}$ of the closed contour $\gamma$

$$
\mathbf{Q}=\int_{\gamma^{*}} d \mathbf{x}=\sum_{i=1}^{n} n_{i} \mathbf{e}_{i}
$$

The latter equality follows from the fact that both ends of curve $\gamma^{*}$ must belong to the lattice $\mathbb{L}$. Note that the same expression can be derived if one use the vectorial structure from (9) in the space of cycles $\gamma$

$$
\mathbf{Q}=\oint_{\gamma} d \phi=\sum n_{i} \mathbf{e}_{i} \oint_{\gamma_{i}} d \phi_{k}=\sum_{i=1}^{n} n_{i} \mathbf{e}_{i}
$$

Further we adopt a second approach, using vectorial structure of homology group, to all considered $\mathcal{M}$, since they will satisfy to conditions of the Gurevich theorem. This choice is very obvious and usually the possible differential forms are fixed by theory action or by symmetry properties.

Thus we have introduced vectorial topological charges of torus $T_{L}$, containing in the explicit form the lattice's basis $\left\{\mathbf{e}_{i}\right\}_{L}$. For obtaining metric and corresponding scalar product in space of topological charges one must consider concrete realization of torus $T_{L}$ and a chiral model on it. Torus $T_{L}$ of rank $n$ can be realized as diagonal $n$-dimensional matrices $\mathbf{t}$

$$
\mathbf{t}=\operatorname{diag}\left(e^{2 \pi i\left(s_{1} \phi\right)}, \ldots, e^{2 \pi i\left(s_{n} \phi\right)}\right)
$$

where () denotes usual scalar product in $n$-dimensional euclidean space $\mathbb{R}^{n}$, and a set $\left\{\mathbf{s}_{i}\right\}$ is a basis of dual lattice $\mathbb{L}^{*}$ from (10-11). It is easy to see that to each elementary cycles $\mathbf{e}_{i}$ corresponds one exponent in $\mathbf{t}$ with dual vector $\mathbf{s}_{i}$.

The lattice $\mathbb{L}^{*}$ is a weight lattice of torus $T_{L}$, considered as a group. For topological interpretation of all weights it is necessary that $\mathbb{L} \supseteq \mathbb{L}^{*}$. For example, a lattice $\mathbb{Z}^{n}$ coincides with its dual one, consequently, all weights of group $T^{n}$ have topological interpretation.

For this reason in a case of general tori $T_{L}$ an important role play tori, corresponding to the self-dual lattices $\mathbb{L}=\mathbb{L}^{*}$, since for them all weights have one to one correspondence with topological charges. But it is not enough for exact isomorphism between them, because one must have the same metric in space of topological charges. As we will see below, it doesn't take place for tori $T_{L}$. 
Now we pass to discussion of the compact chiral models, connected with $T_{L}$ and will describe the explicit realizations of vector topological charges introduced above. The first model generalizes two-dimensional nonlinear $\sigma$ model on a circle $S^{1}$ [19]. We will use for $T_{L}$ a realization in the form (16). One can define two-dimensional nonlinear $\sigma$-model on $T_{L}$ by next action $\mathcal{S}$ (or energy $E$ )

$$
\mathcal{S}=\frac{1}{2 \alpha} \int d^{2} x \operatorname{Tr}\left(\mathbf{t}_{\mu}^{-1} \mathbf{t}_{\mu}\right)=\frac{1}{2 \alpha} \int d^{2} x\left(\phi_{\mu} \cdot \phi_{\mu}\right),
$$

where $\mathbf{t}_{\mu}=\partial_{\mu} \mathbf{t}, \phi_{\mu}=\partial_{\mu} \phi, \mu=1,2$ and here appears new scalar product $(\cdot)$, defined by effective $n$-bein metric $g_{i k}$ on torus space,

$$
(\phi \cdot \phi)=\sum g_{i k} \phi^{i} \phi^{k}, \quad g_{i k}=\sum_{a=1}^{n} s_{i}^{a} s_{k}^{a}
$$

It easy to see that metric $g_{i k}$ for general lattice $\mathbb{L}$ does not coincide (except case of $T^{n}$ ) with euclidean one on $\mathbb{R}^{n}$. Exactly this metric will define interaction of different vector topological charges on torus $T_{L}$.

This chiral model can be considered as effective theory, corresponding in long-wave limit to the Ginzburg-Landau type theories or to discrete lattice type models with vacuum manifold $\mathcal{M}=T_{L}$. Due to nontriviality of $\pi_{1}\left(T_{L}\right)$, it must have vortex-type solutions. These vortex solutions are ill-defined for small scales, where the full action must be used for finding a structure of the vortex core. But for description of the topological properties only longwave behavior will be important and for this reason we shall use at small distances instead of core a short-wave cut-off parameter $a$, which is analog of the lattice constant or of the core radius.

The corresponding equations

$$
\partial^{2} \phi_{i}=0
$$

due to its linearity have $N$-"vortex" solutions in $\mathbb{R}^{2}$ plane with $N$ punctures

$$
\boldsymbol{\phi}(\mathbf{x})=\sum_{i=1}^{N} \mathbf{q}_{i} \frac{1}{\pi} \arctan \left(\frac{y-y_{i}}{x-x_{i}}\right), \mathbf{q}_{i} \in \mathbb{L},\left(q_{i} s_{a}\right) \in \mathbb{Z},(x, y) \in \mathbb{R}^{2}
$$

Energy of one "vortex" with topological charge $\mathbf{q}=\sum_{i=1}^{n} n_{i} \mathbf{e}_{i}$ is logariphmically divergent

$$
E=\frac{(q \cdot q)}{2 \alpha} 2 \pi \ln \frac{R}{a},(q \cdot q)=\sum g_{i k} q^{i} q^{k}=\sum_{i=1}^{n} n_{i}^{2},
$$


where $R$ is a space radius and $a$ is some small size cut-off. It follows from (22) that independently on lattice $\mathbb{L}$ topological charges, corresponding to different cycles, do not interact as in the case of torus $T^{n}$ ! This is a consequence of appearence of effective metric $g_{i k}$ on tori space. Energy of $N$-"vortex" solution $E_{N}$ with whole topological charge $\mathbf{Q}=\sum_{i=1}^{N} \mathbf{q}_{i}=0$ is

$$
E_{N}=\frac{2 \pi}{2 \alpha} \sum_{i \neq k}^{N}\left(\mathbf{q}_{i} \cdot \mathbf{q}_{k}\right) \ln \frac{\left|x_{i}-x_{k}\right|}{a}+C(a) \sum_{i}^{N}\left(\mathbf{q}_{i} \cdot \mathbf{q}_{i}\right),
$$

where scalar product of topological charges is

$$
\left(\mathbf{q}_{i} \cdot \mathbf{q}_{k}\right)=\sum_{a=1}^{n} n_{i}^{a} n_{k}^{a}
$$

and $C(a)$ is some nonuniversal constant, determining "self-energy" (or core energy) of vortices and depending on type of core regularization. Thus, we see that properties of vortex exitations of non-linear $\sigma$-model on all tori $T_{L}$ (with tori dimension equal to their rank, they also include complex abelian tori) are similar to those of non-linear $\sigma$-model, defined on tori $T^{n}$. In particular, the topological charges, corresponding to different cycles, do not interact with each other though they belong to lattice $\mathbb{L} \neq Z^{n}$. It can be understood, since on these tori one can always deform initial cycles into canonical ones. At the same time this result confirms that we have introduced vector structure correctly.

But this is not a whole story. There are tori different from $T^{n}$ and $T_{L}$ types. They can be called degenerated ones because their rank $n$ is smaller than their dimension $p$. We consider them in the next section.

The second model we want to consider here is one-dimensional conformal $\sigma$-model on $T_{L}$, which generalizes analogous $\sigma$-model on $S^{1}$. Its action is defined as

$$
\begin{gathered}
\mathcal{S}=\frac{1}{2 \alpha} \int d x d x^{\prime} \frac{\left|\mathbf{t}(x)-\mathbf{t}\left(x^{\prime}\right)\right|^{2}}{(x-x)^{2}}= \\
\frac{1}{2 \alpha} \int d x d x^{\prime} 2 \sum_{1}^{n}\left(1-\cos \left(s_{i}\left(\phi(x)-\phi\left(x^{\prime}\right)\right)\right)\right.
\end{gathered}
$$

where $x \in \mathbb{R}^{1}$. Following method of paper [16], one can show, that this model has $N$-instanton solutions of the form (20)

$$
\phi(x)=\sum_{i=1}^{N} \mathbf{q}_{i} \frac{1}{\pi} \arctan \frac{x-a_{1 i}}{a_{2 i}}
$$


where $a_{1 i}, a_{2 i}$ are abitrary constants, characterizing place and width of corresponding instanton, and all topological charges $\mathbf{q}_{i}$ in (26) must satisfy simultaneously a condition

$$
\left(q_{i} s_{k}\right) \leq 0 \quad \text { or } \quad\left(q_{i} s_{k}\right) \geq 0
$$

for all $\mathbf{s}_{k}, k=1, \ldots, n$. This condition is a generalization of analogous condition in $\sigma$-model on $S^{1}$, which, in its turn, is an analog of analiticity (or anti-analiticity) property of two-dimensional instantons. The corresponding action is

$$
\mathcal{S}_{N}=\frac{(2 \pi)^{2}}{2 \alpha} \sum_{i=1}^{n}\left|\left(s_{i} Q\right)\right|, \mathbf{Q}=\sum_{i=1}^{N} \mathbf{q}_{i}
$$

Such form of action, linear in $Q$, takes place only for sets of charges, satisfying condition (27). For superpositions with arbitrary charges appear interaction between different charges.

\section{The Cartan tori}

In this section we will be interested in degenerated tori, related with maximal abelian Cartan tori $T_{G}$ of the simple compact Lie groups $G$. We consider their different representations, corresponding homotopical groups $\pi_{1}\left(T_{G}\right)$ and topological charges.

The Cartan torus $T_{G}$ consists of elements

$$
\mathbf{g}=e^{2 \pi i(\mathbf{H} \phi)}, \mathbf{H}=\left\{H_{1}, \ldots, H_{n}\right\} \in \mathfrak{C},\left[H_{i}, H_{j}\right]=0,
$$

where $n$ is a rank of $G, \mathfrak{C}$ is a maximal commutative Cartan subalgebra of the Lie algebra $\mathfrak{G}$ of the group $G$ and we assume that $(H \phi)$ is usual euclidean scalar product. Due to their commutativity all $H_{i}$ can be diagonalized simultaneously. Their eigenvalues are called the weights $\mathbf{w}$ and depend on concrete representation of $G$ and $\mathfrak{C}$. The weights $\left\{\mathbf{w}_{a}\right\}_{\tau}, a=1, \ldots, p$, belonging to a $p$-dimensional irreducible representation $\tau(G)$ form a set "of quantum numbers" of this representation.

All possible weights w of the simply connected group $G$ or of the universal covering groups $\tilde{G}$ of non-simply connected groups $G$ form a lattice of weights $\mathbb{L}_{w}^{G}$. Its basis can be choosed by different ways. The basis, most convenient from the view point of the representation theory, is the so called basis 
of fundamental weights $\overline{\mathbf{w}}_{i}, i=1, \ldots, n$. Any weight $\mathbf{w}$ can be represented in the form

$$
\mathbf{w}=\sum_{1}^{n} n_{i} \overline{\mathbf{w}}_{i}
$$

where all $n_{i}$ are integers. But, in general, not all $\overline{\mathbf{w}}_{i}$ have a minimal norm. In some cases more convenient is a basis of vectors with minimal norm. As in a case of all lattices different basises are related by linear modular transformations $M$ with $\operatorname{det} M= \pm 1$ and integer coefficients [13]. For transformations, preserving an orientation of the basis, $\operatorname{det} M=1$.

The weight lattice $\mathbb{L}_{w}$ and related with it root $\mathbb{L}_{r}$ and dual root $\mathbb{L}_{v}$ lattices are needed for finding $\pi_{1}\left(T_{G}\right)$, which can depend on concrete representation $\tau(G)$. The group $\pi_{1}\left(T_{G}\right)$ can be defined in general form by the methods of the Lie groups and algebras [14], but we will use here more simple and obvious one, analogous to one used above for tori $T_{L}$. Since in any irreducible representation $\tau(G)$ of dimension $p$ one can choose the eigenvectors $\mid a>$ of $\mathbf{H}$ as a basis

$$
\mathbf{H}\left|a>=\mathbf{w}_{a}\right| a>, a=1, \ldots, p,
$$

then in this basis all $H_{i}$ and any element $\mathrm{g} \in T_{G}$ have diagonal form

$$
\mathbf{g}_{\tau}=\operatorname{diag}\left(e^{2 \pi i\left(w_{1} \phi\right)}, \ldots, e^{2 \pi i\left(w_{p} \phi\right)}\right)
$$

Here again $\left(w_{a} \phi\right)$ denotes an usual euclidean scalar product of the vectors $\mathbf{w}_{a}$ and $\phi$. The main difference of this form from usual representation of tori $T_{L}$ type (16) is that:

1) a dimension of diagonal matrices in (32) coincides with dimension of $\tau$-representation $p$, which is usually larger, than rank of $G$,

2) the set of weights $\{\mathbf{w}\}$ has discrete Weyl symmetry, which ensures Weyl invariance of $T_{G}$ and results in the next two properties

$$
\sum_{a=1}^{p} \mathbf{w}_{a}=0, \quad g_{i k}=\sum_{a=1}^{p} w_{i}^{a} w_{k}^{a}=B_{\tau} \delta_{i k},
$$

where constant $B_{\tau}$ depends on representation. It follows from (33) that in case of $T_{G}$ effective metric $g_{i k}$ on the parameter space is proportional to the euclidean one. This fact will appear very important for interaction of the corresponding topological charges and difference of properties of non-linear $\sigma$-models on tori $T_{L}$ and $T_{G}$ [18]. 
From (33) it is obvious, that in this representation all $\mathbf{g} \in T_{G}$ are periodic with a lattice of periods $\mathbb{L}_{\tau}^{-1}$, dual (or inverse) to the lattice $\mathbb{L}_{\tau}$, which is generated by weights $\mathbf{w}_{a}$ of $\tau$-representation. The point is that the lattice $\mathbb{L}_{\tau}$ can be some sublattice of lattice $\mathbb{L}_{w}: \mathbb{L}_{w} \supseteq \mathbb{L}_{\tau}$.

A lattice $\mathbb{L}_{v}$, inverse to $\mathbb{L}_{w}$, is called a lattice of dual roots $\mathbf{r}^{v}$. It has its own basis of the dual roots $\mathbf{r}_{i}^{v}(i=1, \ldots, n)$

$$
\left(r_{i}^{v} w_{k}\right)=\delta_{i k} .
$$

Thus we see that for simply connected $G$ and representations, containing weights, belonging to the $\mathbb{L}_{w}$, but not to some its sublattice, $\pi_{1}\left(T_{G}\right)$ is isomorphic to the $n$-dimensional lattice $\mathbb{L}_{v}$ of the dual roots $\mathbf{r}^{v}$ (see for example [17]). All simple compact groups $G$, except $G=S O(N)$, are simply-connected. For multiply connected $G$ the group $\pi_{1}(G)$ is a subgroup of a finite discrete group - a center $Z_{G}$, which belongs to $\tilde{T}_{G}$ - the maximum torus of a universal covering group $\tilde{G}$. Therefore, for multiply connected $G$ such, that $G=\tilde{G} / \pi_{1}(G)$

$$
\pi_{1}\left(T_{G}\right)=\pi_{1}\left(\tilde{T}_{G}\right) \times \pi_{1}(G)=\mathbb{L}_{v}^{G} \times \pi_{1}(G)
$$

It means, that $\pi_{1}\left(T_{G}\right)$ contains $\mathbb{L}_{v}$ as a sublattice. For groups $S O(N)$ $\pi_{1}(S O(N))=Z_{2}$, and for the adjoint groups $G_{a d}=\tilde{G} / Z_{G}$

$$
\pi_{1}\left(G_{a d}\right)=Z_{G}
$$

For simply-laced groups $G$ (i.e. for groups of a series $A_{n}=S U(n+1), D_{n}=$ $\left.S O(2 n), E=E_{6,7,8}\right) \mathbb{L}_{v}$ coincides with a lattice of all roots $\mathbb{L}_{r}$, which is a sublattice of a lattice of weights $\mathbb{L}_{r} \subseteq \mathbb{L}_{w}$. The structure of all lattices $\mathbb{L}_{w}, \mathbb{L}_{r}, \mathbb{L}_{v}$ of simple compact groups $G$ is known [14]. It is necessary to note, that in general case they are not any more hypercubic and can belong to four different series of $A, D, E, Z$ types. Further we will consider only minimal and adjoint representations. The weights of minimal representations generate a weight lattice $\mathbb{L}_{w}$, thus a lattice of topological charges will be lattice of dual roots $\mathbb{L}_{v}$. The weights of adjoint representation are roots and generate root lattice $\mathbb{L}_{r}$, in this case topological charges will belong to $\mathbb{L}_{r}^{-1}$.

It was shown in [17] that for groups $G=G_{2}, E_{8}, C_{n}, a d A_{n}, a d B_{n}, a d D_{n}$ and $a d E_{6,7}$ the lattice of all possible topological charges $\mathbb{L}_{\tau} \supseteq \mathbb{L}_{w}$, and consequently for them there is a possibility of the topological interpretation of all "quantum numbers" of these groups. There is also possibility of a 
partition $\mathbb{L}_{\tau}^{G}$ into sets of charges, corresponding to the irreducible representations of groups $G^{\prime}$, for which $\mathbb{L}_{\tau}^{G}=\mathbb{L}_{w}^{G^{\prime}}$. For example, $\mathbb{L}_{\tau}^{G_{2}}=\mathbb{L}_{w}^{A_{2}}, \mathbb{L}_{\tau}^{E_{8}}=$ $\mathbb{L}_{w}^{E_{8}}, \mathbb{L}_{\tau}^{C_{n}}=\mathbb{L}_{w}^{C_{n}}$. For other groups $G$, for which $\mathbb{L}_{\tau}^{a d G}=\mathbb{L}_{w}^{G}$ (for example, $\left.G=A_{n}, B_{n}, D_{n}, E_{6,7}\right)$ not all sets of topological charges, answering to some representation (for example, quark representations of groups $A_{n}$ or spinor representations of groups $B_{n}, D_{n}$ ) will correspond to the exact (single-valued) representations.

Now let us consider two-dimensional chiral model on tori $T_{G}$. The corresponding action $\mathcal{S}$ is

$$
\mathcal{S}=\frac{1}{2 \alpha} \int d^{2} x \operatorname{tr}_{\tau}\left(\mathbf{g}_{\nu}^{-1} \mathbf{g}_{\nu}\right)=\frac{1}{2 \alpha} \int d^{2} x t r_{\tau}\left(H \phi_{\nu}\right)^{2}=\frac{1}{2 \alpha} B_{\tau} \int d^{2} x\left(\phi_{\nu}\right)^{2},
$$

where $\mathbf{g}=e^{2 i \pi(H \phi)} \in T_{G}, \quad \phi=\left(\phi_{1}, \ldots, \phi_{n}\right), \quad n$ is a rank of $G, \phi_{\nu}=$ $\partial_{\nu} \phi, \nu=1,2$, and effective metric $g_{i k}$, generated by weight system $\{\mathbf{w}\}_{\tau}$ of $\tau$-representation, from (33) is used. It is convenient to introduce in this model a normalized trace to avoid constant $B_{\tau}$ from effective metric

$$
\operatorname{Tr}=\frac{t r_{\tau}}{B_{\tau}}
$$

Then $\mathcal{S}$ takes a canonical form without coefficient $B_{\tau}$. The corresponding equations of motion

$$
\left(\partial_{\nu}\right)^{2}(H \phi)=0
$$

have classical vortex-type solutions in a region $R>r>a$ analogous to (20)

$$
\boldsymbol{\phi}(\mathbf{x})=\frac{1}{\pi} \mathbf{Q} \arctan \left(\frac{y-y_{0}}{x-x_{0}}\right), \mathbf{Q} \in \mathbb{L}_{\tau}^{-1}
$$

Just these solutions for groups $G$, such, that $\mathbb{L}_{\tau}^{-1} \supseteq \mathbb{L}_{w}$, can give the topological interpretation of all their quantum numbers [17. Energy of these vortices is also logariphmically divergent

$$
E=1 /(2 \alpha) \int\left(\partial_{\mu} \phi\right)^{2} d^{2} x=\frac{1}{2 \alpha}(2 \pi)(\mathbf{Q})^{2} \ln (R / a)
$$

This gives nonzero logariphmic interaction between vortices with different non-collinear topological charges in contrast with a case of tori $T_{L}$

$$
E=\frac{1}{2 \alpha}\left(\mathbf{Q}_{1} \mathbf{Q}_{2}\right) 2 \pi \ln \left|\mathbf{x}_{1}-\mathbf{x}_{2}\right| / a .
$$


This interaction depends on relative orientation of topological charges and for this reason a geometry of lattices becomes very important. Energy of $N$-vortex type solutions will have a form similar to (22) with usual scalar product of topological charges.

Now one can conclude that properties of $\sigma$-models on the Cartan tori will be different and depending on lattice $\mathbb{L}_{\tau}^{-1}$. As was mentioned above, all lattices, connected with compact simple groups, belong to four series $A, D, E, Z$ and in appropriate scales are integer-valued [20]. How they determined critical properties of topological phase transitions is considered in paper [18](see also [15]).

It is interesting to know which spaces, besides tori, can have homotopical group $\pi_{1}$ of lattice type, which must be integer-valued. One must also note, that there are spaces with $\pi_{1}$ having some finite cyclic subgroups, but we do not discuss them here.

One-dimensional conformal $\sigma$-models on tori $T_{G}$ can be analysed as those on tori $T_{L}$. The single difference will be that a sum in trace now goes over all weights of some representation. Since instantons (or anti-instantons) do not interact between them, there will be no essential changes in properties of $\sigma$-models on tori $T_{L}$ and $T_{G}$ as long as we will be interested only unmixed configurations. But they will be different if one consider properties, connected with all possible configurations.

\section{Manifolds with $\pi_{2}=\mathbb{L}$}

Now we pass to consideration of manifolds $\mathcal{M}$ with homotopical group $\pi_{2}$ of lattice type. The more known such manifolds can be divided into two large classes: 1) homogeneous spaces of simple compact Lie groups, 2) the Hodge submanifolds of complex projective spaces $\mathbb{C P}^{n}$, do not coinciding with $\mathcal{M}$ from 1 ). The first ones are the more interesting from the point of view, accepted in this paper because they can have $\pi_{2}$ group with topological charges belonging to the weight lattices (or their sublattices) of the corresponding groups. For finding out instanton type solutions with topological charges $\mathbf{q} \in \mathbb{L}_{r}$ the more important are the complex homogeneous spaces. The main classification theorem about such manifolds says that all of them can be considered as bundle spaces over some flag spaces $F$

$$
\mathcal{M} \stackrel{T}{\rightarrow} F
$$

where a fiber $T$ is a parallelizable space. Moreover $T$ must be some complex torus if $\mathcal{M}$ is simply connected or if $\mathcal{M}$ is kahlerian. If both these conditions 
take place, then $\mathcal{M}$ must be some flag space $F\left[21\right.$, 22]. Since $\pi_{2}(T)=0$, the most important from instantons point of view are the flag spaces $F$. Among them there are maximal flag spaces $F_{G}=G / T_{G}$ with $\pi_{2}\left(F_{G}\right)=\mathbb{L}_{v}$ [17, 23.

The chiral models on $F_{G}$ were considered in papers [23, 24], where it was shown, that since $\pi_{2}\left(G / T_{G}\right)=\pi_{1}\left(\tilde{T}_{G}\right) \neq 0$ and on $G / T_{G}$ there is complex structure, the appropriate equations have holomorphic instanton solutions. But their consideration of topological charges was carried out not taking into account vectorial structure of corresponding group $\pi_{2}\left(F_{G}\right)$. The authors have proceeded from the assumption, that appropriate topological charges are simply integer numbers, corresponding to convolution of some 2 -form with different 2-subspaces. Since $\pi_{2}\left(G / T_{G}\right)=\mathbb{L}_{v}$, it is possible to consider corresponding topological charges as isovectors. To see how they can be realized, let us return to $\sigma$-model on $G / T_{G}$. Its action has the form 23

$$
\mathcal{S}[u]=\frac{1}{2 g^{2}} \int d^{2} x g_{a \bar{b}}(u, \bar{u}) \partial_{\mu} u^{a} \partial_{\mu} \bar{u}^{b}, a, b=1, \ldots, p, 2 p+n=\operatorname{dim} G .
$$

Here $u^{a}$ are complex local coordinates on $G / T_{G}$, and the metric $g_{a \bar{b}}(u, \bar{u})$ is a representative of the 2 -forms from class $\varrho$, where $\varrho$ is an isovector, equal to a half-sum of all positive roots of Lie algebra $\mathcal{G}$

$$
\varrho=\frac{1}{2} \sum_{a>0} \mathbf{r}_{a}=\sum_{i=1}^{n} \bar{\omega}_{i}
$$

here $\bar{\omega}_{i}$ are the fundamental weights of group $G$. Just this metric is the Einstein one on $G / T_{G}$ 24, 25], i.e.

$$
g_{a \bar{b}}(u, \bar{u})=k R_{a \bar{b}}(u, \bar{u}), k=\frac{1}{2},
$$

where $R_{a \bar{b}}(u, \bar{u})$ is the Ricci tensor. Such choice of invariant metric ensures a renormalizability of (42) 24, 26.

On holomorphic fields $u^{a}(z), z \in \mathbb{C}=\mathbb{R}^{2}$, which are the coordinates on $F_{G}$, action $\mathcal{S}$ formally coincides (up to coefficient) with topological invariant

$$
Q=\frac{1}{2} \int d^{2} x g_{a \bar{b}}(u, \bar{u}) \varepsilon_{\mu \nu} \partial_{\mu} u^{a} \partial_{\nu} \bar{u}^{b}=\frac{1}{2} \int g_{a \bar{b}} d u^{a} \wedge d \overline{u^{b}}
$$


As is known [21, 24], all integer-valued 2-forms on $F_{G}=G / T_{G}$

$$
\Omega=\Omega_{a \bar{b}} d u^{a} \wedge d \bar{u}^{b}
$$

can be decomposed (modulo exact forms) on basis of the 2-forms $\omega_{i}, i=$ $1, \ldots, n$, parametrized by fundamental weights $\bar{\omega}_{i}$

$$
\Omega=\sum_{1}^{n} c_{i} \omega_{i}, c_{i} \in \mathbb{Z} .
$$

Similar expansion with coefficients $c_{i}=1$ has the 2-form corresponding to the metric $g_{a \bar{b}}(u, \bar{u})$. On the other hand on $G / T_{G}$ there is a space of 2 -cycles $\gamma$, which is dual to space of the 2-forms. The 2-cycles are given by linear combinations of the Shubert cells of minimum dimension: real dimensionality 2 or complex dimensionality 1 , number of which is equal to a rank of $G$ [21]. Since $\pi_{1}\left(F_{G}\right)=0$ and according to the Gurevich theorem $\pi_{2}\left(F_{G}\right)=H_{2}\left(F_{G}, \mathbb{Z}\right)=\mathbb{L}_{v}$, it is natural to introduce on the space of 2-cycles a vectorial structure similar to (9) and to choose a parametrization of 2-cycles by a lattice $\mathbb{L}_{v}$, then its basic cycles $\left\{\gamma_{i}\right\}$ are parametrized by the simple dual roots $\mathbf{r}_{i}^{v}$

$$
\gamma \rightarrow \gamma=\sum_{1}^{n} n_{i} \gamma_{i} \mathbf{r}_{i}^{v}, n_{i} \in \mathbb{Z} .
$$

A convolution of the 2-forms $\omega_{i}$ and of dual to them 2-cycles $\gamma_{k}$, i.e. integral of the 2 -form $\omega_{i}$ over a 2 -cycle $\gamma_{k}$ is equal

$$
\left(\omega_{i} \circ \gamma_{k}\right)=\int_{\gamma_{k}} \omega_{i}=\delta_{i k}
$$

Since 2-form $\varrho$, determining topological charges, is fixed, the vectorness of space of 2-cycles induces a vectorness of space of topological charges $\mathbf{Q}$

$$
\mathbf{Q}=\sum_{i=1}^{n} q_{i} \mathbf{r}_{i}^{v}=\left(\varrho \circ \sum_{1}^{n} q_{i} \gamma_{i}\right) \mathbf{r}_{i}^{v}, q_{i} \in \mathbb{Z}
$$

From this it follows that topological charges, answering to different independent cycles, cannot be simply added and, in particular, compensate each other. For evaluations it is useful to note similarity of topological cell-like structure of torus $T_{G}$ and flag $G / T_{G}$ in their lowest nontrivial cell complexes: for a torus $T^{n}$ it is a bouquet of circles $T^{n}=S_{1}^{1} \vee \ldots \vee S_{1}^{1}$ [13] and for a flag space it will be an analog of bouquet of 2-spheres $M=S_{1}^{2} \vee \ldots \vee S_{1}^{2}$. 
For example, for $G=S U(n+1)$ as local coordinates on these 2-spheres it is possible to choose the elements of upper, nearest to a main diagonal, diagonal line. Then, corresponding to these 2-spheres instantons are simply instantons of Belavin - Polyakov [4]. For an action $\mathcal{S}$ of $n$ instantons with topological charges $q_{1}, \ldots, q_{n}$, corresponding to $n$ different 2 -cycles, we shall receive

$$
\mathcal{S}=\frac{1}{2 g^{2}} \sum_{1}^{n}\left|q_{i}\right|
$$

This expression is similar to that for one-dimensional instantons from (28).

Thus it is shown, that instanton solutions of two-dimensional chiral theories on maximal flag spaces $F_{G}=G / T_{G}$ can realize isovectorial topological charges, belonging to $\pi_{2}\left(G / T_{G}\right)=\mathbb{L}_{v}$, and therefore can realize nearly all quantum numbers of the appropriate group $G$. But, as in one-dimensional case, instantons do not interact with each other. For this reason, only instantons cannot realize real particles. For their realization one need exitations, interacting like vortices, but with Coulomb or Yukava type potential $V(r)$

$$
E=\left(\mathbf{q}_{1} \mathbf{q}_{2}\right) V(r), V_{C}(r)=\frac{1}{r}, V_{Y}(r)=\frac{e^{-m r}}{r}
$$

Such type of interaction can be obtained due to gauge fields, which appear naturally when one consider $T_{G}$ and $F_{G}$ as submanifolds in $G$ and takes into account the fact that in $G$ there is a whole set of such conjugated submanifolds. Then, if there is no fixing potential, all these submanifolds will be on equal foot. It means that corresponding $\sigma$-models fields, taking their values in these submanifolds, at different points of physical space can take values in different submanifolds and conjugation transformations will play a role of gauge fields. A work in this direction is now going on.

\section{3-dimensional topological exitations}

Topological exitations of vortex and instanton types, i.e., connected with $\pi_{1}\left(T_{G}\right)$ and $\pi_{2}\left(F_{G}\right)$, can exist also in three-dimensional space. Vortex type exitations will form now closed or open lines, in a latter case its energy will be $E \sim L \ln R / a$, where $L$ is a line's length. Topological exitations connected with topological charges $\mathbf{Q} \in \pi_{2}\left(G / T_{G}\right)$ can exist in the generalized Higgs-Salam-Weinberg models as 3-dimensional particle-like monopoles. They will have finite energy if corresponding gauge field would be engaged 
and will have divergent energy without gauge field. It is interesting note that since $\pi_{2}\left(F_{G}\right)=\mathbb{L}_{v}$, which in $G=S U(n)$ case does not contain weights of the fundamental quark representations, the latter cannot exist as topological exitations on $F_{G}$. This fact demands additional factorization of $F_{G}$ or spontaneous symmetry breaking if one want to get topological interpretation of quarks also (see for example [27]).

Here we discuss as an example instantons exitations connected with homotopic group $\pi_{2}$ in 3-dimensional chiral model on sphere $S^{2}$ with action

$$
S=\frac{1}{2 \alpha} \int d^{3} x\left(\partial_{\mu} \mathbf{n}\right)^{2}, \mathbf{n} \in S^{2}
$$

The corresponding equations have instanton type solution, which we represent in a form analogous (39)

$$
n_{i}(x)=\frac{x_{i}}{r} \theta_{r}(r-a),
$$

where $\theta_{r}(r)$ is a regularized step-function. Its topological charge

$$
Q=\frac{1}{4 \pi} \int d S
$$

where integral $\int d S$ is over sphere in internal space, and energy

$$
E=\frac{1}{2 \alpha} \int d^{3} x\left(\partial_{\mu} \mathbf{n}\right)^{2}=\frac{1}{\alpha} 4 \pi \int_{a}^{R} d r \simeq \frac{4 \pi}{\alpha} R .
$$

grows linearly with $R$. It means that in this model instantons must be confined in neutral pairs like quarks in QCD and will appear themselves only at small distancies! All infra-red properties will not depend on their existence. Analogous properties will have $\sigma$-models on other manifolds having the same homotopical group $\pi_{2}=\mathbb{Z}$ or of vectorlike type $\pi_{2}=\mathbb{L}$ as in a case of $F_{G}$.

One can show (for example [14]), using exact homotopic sequence for bundle $G \stackrel{T_{G}}{\longrightarrow} F_{G}$ and the fact, that $\pi_{i}\left(T_{G}\right)=0, i>1$,

$$
\pi_{k}(G)=\pi_{k}\left(G / T_{G}\right)=\pi_{k}\left(G, T_{G}\right), k=3,4 \ldots
$$

This means, that for $k>2$, the flag space $F_{G}$ has the same homotopical groups as group $G$ itself. In particular, in a case of 3-dimensional non-linear 
$\sigma$-models on flag spaces $F_{G}$ they, probably, can have knotted configurations, connected with group $\pi_{3}\left(F_{G}\right)=\pi_{3}(G)=\mathbb{Z}$, similar to those proposed recently for 4-dimensional Yang-Mills theory in [28].

All this facts about reach set of topological exitations on Cartan tori $T_{G}$ and flag spaces $F_{G}$ say that manifolds $T_{G}$ and $F_{G}$ and theories on them or their gauge generalizations could be considered as necessary ingredient of any high energy GUT-type or string-type theories, which try to obtain the obvious physical (in particular, topological) interpretation of elementary particles.

The author is grateful to Prof. G.Volovik for useful discussion of some problems, considered in this paper, and to Prof. V.Gurarie for kind information about literature on complex homogeneous spaces.

The work was supported by RFBR grants 96-02-17331-a and 96-1596861.

\section{References}

[1] A.M.Polyakov, JETP Letters 20 (1974) 430.

[2] G.t'Hooft, Nucl.Phys. B79 (1974) 276.

[3] A.A.Belavin, A.M.Polyakov, A.S.Schwartz, Yu.S.Tyupkin, Phys.Lett. B59 (1975) 85.

[4] A.A.Belavin, A.M.Polyakov, JETP Letters 22 (1975) 245.

[5] D.Mattis, The Theory of Magnetism.Harper\& Row Publishers, 1965.

[6] W.H.Thomson, Trans.Roy.Soc.Edin. 25, (1869) 217.

[7] P.G.Tait, On knots, I,II,III, Scientific Papers, Cambridge University Press, 1900; M.F.Atiyah, The Geometry and Physics of Knots. Cambridge University Press, 1990.

[8] L.D.Landau, E.M.Lifshitz, The Hydrodynamics. Nauka, Moscow, 1986.

[9] P.G.De Gennes, Superconductivity of Metals and Alloys, W.A.Benjamin, Inc.,1966.

[10] M.D.Frank-Kamenetskii, A.V.Lukashin, A.V.Vologodskii, Nature 258 (1975) 398; M.D.Frank-Kamenetskii, A.V.Vologodskii, Uspehi Fiz. Nauk 134 (1981) 641. 
[11] G.E.Volovik, Exotic properties of superfluid ${ }^{3} \mathrm{He}$. World Scientific, Singapore, 1992.

[12] R.Rajaraman, Solitons and instantons, North-Holland Pub.Company, Amsterdam-New-York-Oxford, 1982.

[13] B.A.Dubrovin, S.P.Novikov, A.T.Fomenko, Modern geometry,part I, II. Moscow, 1979; part III, Moscow, 1984.

[14] N.Bourbaki, Lie groups and Lie algebras, Mir, Moscow, 1972, 1978.

N.Bourbaki, Groupes et algebres de Lie. Chapters IV-VI, Hermann, Paris, 1968; Chapters VII,VIII, Hermann, Paris, 1975).

[15] S.A.Bulgadaev, Nucl.Phys.B224 (1983) 349; Phys.Lett.B166 (1986) 88.

[16] S.A.Bulgadaev, Phys.Lett. A125 (1987) 299.

[17] S.A.Bulgadaev, JETP Letters, 63 (1996) 796.

[18] S.A.Bulgadaev, JETP Letters, 63 (1996) 780.

[19] A.M.Polyakov, Gauge Fields and Strings. Harwood Academic Publishers, 1987.

[20] G.H.Conway, N.J.A.Sloane, Sphere Packing, Lattices and Groups, vol.I,II. Springer-Verlag, 1988.

[21] N.E.Hurt, Geometric Quantization in Action. D.Reidel Pub.Company, 1983.

[22] S.Kobayasi, K.Nomidzu, Foundations of Differential Geometry. vol.II. Interscience Publishers, 1969.

[23] A.M.Perelomov, Usp.Fiz.Nauk 134 (1981) 577.

[24] A.M.Perelomov, M.C.Prati, Nucl.Phys. B258 (1985) 647.

[25] D.S.Freed, "Flag Manifolds and Infinite Dimensional Kahler Geometry" in Proceedings of International Conference, 1985.

[26] E.Witten, Phys.Rev. D16, 2991 (1977).

[27] T.Vachaspati, Phys.Rev.Lett. 76, 188 (1996). 
[28] L.D.Faddeev, A.Niemi, Toroidal configurations as stable solitons. hepth/9705176. 